\title{
The virial theorem in refined Thomas-Fermi-Dirac theory for the interior of atoms in a solid
}

\author{
Zhu Ru-Zeng(朱如曾) $)^{\mathrm{a})}$, Wen Yu-Hua(文玉华) ${ }^{\mathrm{b})}$, and Qian Jin(钱 劲) ${ }^{\mathrm{a})}$ \\ a) Institute of Mechanics, Chinese Academy of Sciences, Beijing 100080, China \\ b) Department of Physics, Jinhua University, Beijing 100084, China
}

(Received 28 March 2002; revised manuscript received 15 May 2002)

The virial theorem in refined Thomas-Fermi-Dirac theory for the interior of atoms in a solid is given and proven.

Keywords: refined Thomas-Fermi-Dirac theory, virial theorem

PACC: 6400,6410

\section{Introduction}

For the structures and properties of nanomaterials, and the behaviours of micro- and nanosystems, the quantum effects are known to be of more or less importance. The semi-classical ThomasFermi-Dirac (TFD) theory is one of those theories simplified to treat the quantum effects. In TFD theory, there is no quantum tunnelling effect, i.e. there is no possibility of electrons being detected in the areas where the potential is higher than the energy of the electrons. In the 1990s, Cheng and co-workers proposed the refined TFD theory, ${ }^{[1-5]}$ in which the quantum tunnelling effect for atoms in a solid is included by a pseudo-potential for the system. Cheng and Cheng ${ }^{[6]}$ and Chen et $a^{[7]}$ have shown that the pseudo-potential will affect the properties of materials, so that to discuss this theory and its deductions in detail will be useful for the study of the structures and properties of nano-materials, and the behaviours of micro- and nano-systems, as well as the structures and some properties of some materials and their surfaces.

In Ref.[8], the important virial theorems for the electrons on the atomic surface and for the whole atom in the refined TFD theory have been proven. In the present paper, the virial theorem for the interior of the atom in the same theory is given.

\section{The proof of the virial theorem in refined TFD theory for the interior of atoms in a solid}

According to Ref.[8], consider an atom in a solid in the basic state as a Fermi ball with radius $R$ having the surface charge density $\sigma=Q / 4 \pi R^{2}$ on its surface $S(R)$, contributed by the tunnel electrons belonging to this atom, where $Q$ is the total charge of the tunnel electrons.

Let $p(r)$ be the pressure at $r$ from the nucleus. To deduct $p(r)$ at $r<R$, we draw a surface $S(r)$ with radius $r$ and the centre at the nucleus. The total energy $E$ of the system can be written as

$$
E=E_{\text {in }}+E_{\text {out }}+E_{\text {i-o }}+\Phi,
$$

where $E_{\text {in }}, E_{\text {out }}, E_{\mathrm{i}-\mathrm{o}}$ and $\Phi$ are respectively the energy of the part inside $S(r)$ including the nucleus, that of the part outside $S(r)$ except the surface charge, the interaction energy between the two parts, and the pseudo-potential, which includes the self-energy of the surface charge and the interaction energy between it and both of the nucleus and all the volume charges. We have

$$
E_{\text {in }}=\int_{r_{1}<r}\left(E_{\mathrm{k}}+E_{\text {cin }}+E_{\text {ex }}\right) \mathrm{d} V_{1},
$$

\footnotetext{
${ }^{*}$ Project supported by the National Natural Science Foundation of China (Grant No 10172088).
} 


$$
\begin{gathered}
E_{\text {out }}=\int_{r<r_{1}<R}\left(E_{\mathrm{k}}+E_{\text {cout }}+E_{\text {ex }}\right) \mathrm{d} V_{1}, \\
E_{\mathrm{i}-\mathrm{o}}=-\int_{r}^{R} \phi\left(r_{1}\right) \operatorname{en}\left(r_{1}\right) \mathrm{d} V_{1}, \\
\Phi=-Q^{2} / 2 R,
\end{gathered}
$$

where $e$ is the absolute value of the charge of an electron, $n\left(r_{1}\right)$ is the density of electrons at distance $r_{1}$ from the nucleus, $E_{\mathrm{k}}\left(r_{1}\right)$ is the density of kinetic energy of electrons at $r_{1}$ from nucleus, $E_{\text {cin }}\left(r_{1}\right)$ is that of Coulomb energy provided by all the charged particles inside $S(r), E_{\text {cout }}\left(r_{1}\right)$ is that of Coulomb energy provided by all the charged particles outside $S(r)$ except the surface charge, $E_{\text {ex }}\left(r_{1}\right)$ is that of the exchange energy among electrons, and $\phi\left(r_{1}\right)$ is the potential at $r_{1}$ provided by all the charges inside $S(r)$. Eq.(5) can be found in Ref.[8]. We have

$$
E_{\mathrm{k}}\left(r_{1}\right)=\left(3 h^{2} / 10 m\right)(3 / 8 \pi)^{2 / 3} n\left(r_{1}\right)^{5 / 3},
$$

$$
\begin{gathered}
E_{\text {cin }}\left(r_{1}\right)=-\left(Z e^{2} / r_{1}\right) n\left(r_{1}\right)+(1 / 2) \\
\times \int_{r_{2}<r}\left[n\left(r_{1}\right) n\left(r_{2}\right) e^{2} / r_{12}\right] \mathrm{d} V_{2}, \quad\left(r_{1}<r\right)(7) \\
E_{\text {cout }}\left(r_{1}\right)=(1 / 2) \int_{r_{2}>r}\left[n\left(r_{1}\right) n\left(r_{2}\right) e^{2} / r_{12}\right] \mathrm{d} V_{2}, \\
\left(r_{1}>r\right) \\
E_{\text {ex }}\left(r_{1}\right)=-(3 / 4) e^{2}(3 / \pi)^{1 / 3} n\left(r_{1}\right)^{4 / 3},
\end{gathered}
$$

where $r_{12}$ is the distance between two electrons at $\boldsymbol{r}_{1}$ and $\boldsymbol{r}_{2}, Z$ is the number of the nuclear charges, and $m$ is the mass of an electron. Eqs.(6) and (9) can also be found in Ref.[8].

In order to obtain the virial theorem for the interior of the atom under the refined TFD theory, we reduce $S(r)$ to $S(r+\mathrm{d} r)$ slowly with the volume density and surface density of the electrons outside $S(r)$ remaining unchanged, while the electrons inside $S(r)$ change adiabatically so as to be kept inside $S(r+\mathrm{d} r)$. For this process, we have

$$
(\mathrm{d} E)_{\mathrm{ad}}=-p(r)(\mathrm{d} V(r))_{\mathrm{ad}},
$$

where $V(r)$ is the volume included by $S(r)$ and the subscript "ad" denotes adiabatic. Obviously we have $\mathrm{d} E_{\text {out }}=0$, and because of the spherical symmetry and according to the static electricity theorem, we have $\mathrm{d} E_{\mathrm{i}-\mathrm{o}}=\mathrm{d} \Phi=0$. So Eqs.(1) and (10) give

$$
p(r)=-\left(\mathrm{d} E_{\text {in }} / \mathrm{d} V(r)\right)_{\mathrm{ad}} .
$$

By the application of Eqs.(2) and (11), we have

$$
\begin{aligned}
p(r)= & -\left\{\left[E_{\mathrm{k}}(r)+E_{\text {ex }}(r)-e n(r) \phi(r)\right]\right. \\
& +\int_{r_{1}<r}\left[\left(\partial\left(E_{\mathrm{k}}+E_{\text {ex }}+E_{\text {cin }}\right) / \partial n\right)\right. \\
& \left.\left.\times\left(\partial n\left(r_{1}\right) / \partial V(r)\right)_{\mathrm{ad}} \mathrm{d} V\left(r_{1}\right)\right]\right\} .
\end{aligned}
$$

On the other hand, for the basic state under consideration, the energy functional (1) should take the least value under the restriction condition of the total number of the electrons inside the surface $S(R)$

$$
N=\int_{r_{1}<R} n\left(r_{1}\right) \mathrm{d} V_{1}
$$

being constant, so that the functional with Lagrangian multiplier $\lambda$

$$
L=E+\lambda \int_{r_{1}<R} n\left(r_{1}\right) \mathrm{d} V_{1}
$$

should take the least value for the function $n\left(r_{1}\right)$. The condition of variation of the functional $L$ by $n\left(r_{1}\right)$ being zero gives the equation

$$
\partial\left(E_{\mathrm{k}}\left(r_{1}\right)+E_{\mathrm{ex}}\left(r_{1}\right)+E_{\mathrm{cin}}\left(r_{1}\right)\right) / \partial n\left(r_{1}\right)=-\lambda .
$$

Substituting Eq.(15) into Eq.(12), we obtain

$$
\begin{aligned}
p(r)= & -\left\{\left[E_{\mathrm{k}}(r)+E_{\mathrm{ex}}(r)-e n(r) \phi(r)\right]\right. \\
& \left.\left.-\lambda \int_{r_{1}<r}\left(\partial n\left(r_{1}\right) / \partial V(r)\right)_{\mathrm{ad}} \mathrm{d} V\left(r_{1}\right)\right]\right\} .
\end{aligned}
$$

The conservation of the number of the electrons inside the surface $S(r)$ gives

$$
\begin{aligned}
0 & =\partial\left(\int_{r_{1}<r} n\left(r_{1}\right) \mathrm{d} V\right) / \partial V(r) \\
& =n(r)+\int_{r_{1}<r}\left(\partial n\left(r_{1}\right) / \partial V(r)\right) \mathrm{d} V\left(r_{1}\right) .
\end{aligned}
$$

Using Eq.(17), Eq.(16) becomes

$$
p(r)=-\left\{E_{\mathrm{k}}(r)+E_{\mathrm{ex}}(r)-e n(r) \phi(r)+\lambda n(r)\right\} .
$$


From Eqs.(6), (7), (9) and (15), we obtain

$$
\lambda n(r)=-(5 / 3) E_{\mathrm{k}}(r)-(4 / 3) E_{\mathrm{ex}}(r)+e n(r) \phi(r) .(19)
$$

Substituting Eq.(19) into Eq.(18) gives the virial theorem for the interior of the atom in a solid

$$
p(r)=(1 / 3)\left[2 E_{\mathrm{k}}(r)+E_{\mathrm{ex}}(r)\right],
$$

which differs from Eq.(13) in Ref.[8] by a term $\Phi_{0} /\left(4 \pi R^{3}\right)$.

\section{References}

[1] Cheng K J and Cheng S Y 1993 Sci. Nature Prog. 3211

[2] Cheng S Y and Cheng K J 1993 Acta Phys. Sin. (Overseas Edition) 2439

[3] Cheng K J and Cheng S Y 1990 Proc. CMRS International Symposium p783

[4] Cheng K J and Cheng S Y 1991 Adv. Mech. (Special for Physical Mechanics) 2123 (in Chinese)
[5] Cheng K J, Fan Q K and Gao Z P 1984 Acta Phys. Sin. 33176 (in Chinese)

[6] Cheng K J and Cheng S Y 1996 Chin. J. Mater. Res. 18 1 (in Chinese)

[7] Chen Q F, Sun Z H, Cai L C and Jing F Q 2001 Chin. Phys. 101144

[8] Cheng K J and Cheng S Y 1995 Acta Phys. Sin. (Overseas Edition) 4344 